\title{
KAZHDAN CONSTANTS FOR COMPACT GROUPS
}

\author{
MARKUS NEUHAUSER
}

(Received 7 June 2004; revised 9 February 2005)

Communicated by A. H. Dooley

\begin{abstract}
It is shown that for the computation of the Kazhdan constant for a compact group only the regular representation restricted to the orthogonal complement of the constant functions needs to be taken into account.
\end{abstract}

2000 Mathematics subject classification: primary 22C05; secondary 22D10.

Kazhdan constants are a quantitative version of property $\mathrm{T}$, which was introduced by Kazhdan [8] in 1967. This property is representation theoretic with remarkable applications, see [7] for an account. The related constants yield a sort of distance between the trivial representation and those not containing it. The question of calculating Kazhdan constants appears as a natural question in [7, page 133]. Explicit Kazhdan constants can be useful, for example, in connection with expanding graphs [9], random walks [12], or the product replacement algorithm [10].

Although it is an easy observation that a compact group has property $\mathrm{T}$, the computation of Kazhdan constants is nevertheless not trivial even for this class of groups, compare with, for example, $[1-4,11]$. The purpose of the theorem in this note is to facilitate in some sense further computations of Kazhdan constants for compact groups.

Let $G$ be a locally compact group. For a subset $Q$ of $G$ and a strongly continuous unitary representation $\pi$ of $G$ on the representation space $H_{\pi}$, let

$$
\kappa_{G}(Q, \pi)=\inf _{\xi \in S_{\pi}} \sup _{g \in Q}\|\pi(g) \xi-\xi\|
$$

Supported by grant 20-65060.01 of the Swiss National Fund for Scientific Research and by FWF (Austrian Science Fund) project P15577.

(C) 2006 Australian Mathematical Society 1446-7887/06 $\$$ A2.00+0.00 
where $S_{\pi}=\left\{\xi \in H_{\pi}:\|\xi\|=1\right\}$ is the unit sphere in $H_{\pi}$. The Kazhdan constant is defined by $\kappa_{G}(Q)=\inf _{\pi \in r(G)} \kappa_{G}(Q, \pi)$, where $r(G)$ is the set of all equivalence classes of representations of $G$ on separable Hilbert spaces not containing the trivial representation. Another constant depending only on the irreducible representations can be defined by $\hat{\kappa}_{G}(Q)=\inf _{\pi \in \widehat{G} \backslash\{1\}} \kappa_{G}(Q, \pi)$, where $\widehat{G}$ denotes the set of equivalence classes of irreducible representations of $G$.

Note that if $\sigma$ is a subrepresentation of $\pi$ then $\kappa_{G}(Q, \pi) \leq \kappa_{G}(Q, \sigma)$. Let $m \in$ $\mathbb{N} \cup\{\infty\}$, and denote by $m \pi$ the $m$-fold direct sum of the representation $\pi$ on $H_{\pi}^{m}$. Then in general only $\kappa_{G}(Q, m \pi) \leq \kappa_{G}(Q, \pi)$, but equality need not hold necessarily. An explicit example where equality does not hold is given in [11]. There, $G=\operatorname{SU}(2), Q$ is any conjugacy class of a non-central element and $\pi_{2}$ is the unique (up to equivalence) irreducible representation of degree 3 . In this case $\kappa_{G}\left(Q, \pi_{2}\right)>\kappa_{G}\left(Q, 3 \pi_{2}\right)$.

Let now $G$ be compact and denote by $L_{0}^{2}(G)$ the orthogonal complement of the constant functions in $L^{2}(G)$ where the compact group $G$ is naturally equipped with the unique normalised Haar measure. Let $\rho$ be the regular representation of $G$ restricted to $L_{0}^{2}(G)$. Obviously $\kappa_{G}(Q) \leq \kappa_{G}(Q, \rho) \leq \hat{\kappa}_{G}(Q)$ holds in general. An easy consequence of the Peter-Weil theorem, see, for example, [6, page 133], is $\kappa_{G}(Q)=\kappa_{G}(Q, \infty \rho)$. The following result, which will be proven below, states in fact that $\infty$ can be omitted.

THEOREM. Let $\rho$ be the regular representation of the compact group $G$ restricted to $L_{0}^{2}(G)$ and $Q$ a subset of $G$. Then $\kappa_{G}(Q)=\kappa_{G}(Q, \rho)$.

For special cases of $G$ and $Q$, this appears in $[1-4,11]$.

To be more precise, [2] states that the result holds in the special case of the dihedral group $G=D_{n}=\left\langle a, b: a^{2}, b^{2},(a b)^{n}\right\rangle$ and $Q=\{a, b\}$, as well as for any abelian compact group $G$ with a compact generating set $Q$. In the first case also $\kappa_{G}(Q)=\hat{\kappa}_{G}(Q)$. The result for abelian $G$ appears likewise in [3, page 463]. For the instance, where $G$ is the cyclic group of order $n$ and $Q=G$, the result can be found again in [1] and furthermore for $G$ the symmetric group and $Q=$ $\{(1,2),(2,3), \ldots,(n-1, n)\}$. Note also that in the first case $\kappa_{G}(Q)<\hat{\kappa}_{G}(Q)$ if $n \geq 4$ while in the second $\kappa_{G}(Q)=\hat{\kappa}_{G}(Q)$. Moreover, in the latter case the Kazhdan constant is equal to $\kappa_{G}(Q, \pi)$ where $\pi$ is the irreducible representation corresponding to the natural action of $S_{n}$ on $\mathbf{C}^{n}$, that is, the representation corresponding to the partition $(n-1,1)$. It is observed in [1, page 496] that $\kappa_{G}(Q)=\hat{\kappa}_{G}(Q)$ or $\kappa_{G}(Q)<\hat{\kappa}_{G}(Q)$ really depends not only on $G$ but also on $Q$. For any compact group $G$ with $Q=G$ the theorem is included in [4, page 309]. It is contained in [11] for any compact group $G$ and $Q$ a conjugacy class.

For the proof of the theorem note that by definition $\kappa_{G}(Q) \leq \kappa_{G}(Q, \rho)$. Hence it suffices to demonstrate that $\kappa_{G}(Q, \rho) \leq \kappa_{G}(Q, \pi)$ for any $\pi \in r(G)$. As noted before, by the Peter-Weil theorem a restriction to the case $\pi=\infty \rho$ would be possible. 
However, this would not significantly simplify the proof presented below.

PROOF. Let $\pi$ be a representation of the compact group $G$ not containing the trivial representation and $\xi \in H_{\pi}$. Then the function $g \mapsto\langle\pi(g) \xi$, $\xi\rangle$ is continuous, and thus square-integrable, as $G$ is compact. By [5, page 309] and the fact that $\pi$ does not contain the trivial representation, there exists an $f \in L_{0}^{2}(G)$ such that $\langle\pi(g) \xi, \xi\rangle=\langle\rho(g) f, f\rangle$ for all $g \in G$. Then $\|\xi\|=\|f\|$ can be read off for $g=1$. Hence

$$
\begin{aligned}
\|\pi(g) \xi-\xi\|^{2} & =2\|\xi\|^{2}-2 \operatorname{Re}\langle\pi(g) \xi, \xi\rangle \\
& =2\|f\|^{2}-2 \operatorname{Re}\langle\rho(g) f, f\rangle \\
& =\|\rho(g) f-f\|^{2} .
\end{aligned}
$$

Thus

$$
\kappa_{G}(Q, \pi)=\inf _{\xi \in S_{\pi}} \sup _{g \in Q}\|\pi(g) \xi-\xi\| \geq \inf _{f \in S_{\rho}} \sup _{g \in Q}\|\rho(g) f-f\|=\kappa_{G}(Q, \rho),
$$

and this proves the theorem.

Finally note that in general the statement of the theorem does not hold for noncompact locally compact groups as for example a compactly generated group which is not amenable and does not have property $\mathrm{T}$ with a compact generating set $Q$ satisfies $\kappa_{G}(Q)=0<\kappa_{G}(Q, \rho)$. A specific example would be the free group on two generators. Here, of course, $\rho$ is just the regular representation as $L_{0}^{2}(G)=L^{2}(G)$ because there are no non-zero constant functions.

A remark pointed out by A. Żuk is that the theorem also holds for non-compact amenable groups $G$ and compact subsets $Q$, since both constants are then 0 . Even more generally, this holds for any subset $Q$ of $G$, since for an amenable group $G$ any representation of $G$ is weakly contained in the regular representation see, for example, [5, page 358] which implies $\kappa_{G}(Q, \rho) \leq \kappa_{G}(Q)$.

\section{Acknowledgements}

It is a pleasure to thank A. Valette for his comments and suggestions and B. Bekka for reference [5].

\section{References}

[1] R. Bacher and P. de la Harpe, 'Exact values of Kazhdan constants for some finite groups', $J$. Algebra 163 (1994), 495-515. 
[2] A. Deutsch, Kazhdan's property ( $T)$ and related properties of locally compact and discrete groups (Ph.D. Thesis, University of Edinburgh, 1992).

[3] _ 'Kazhdan constants for the circle', Bull. London Math. Soc. 26 (1994), 459-464.

[4] A. Deutsch and A. Valette, 'On diameters of orbits of compact groups in unitary representations', J. Austral. Math. Soc. (Series A) 59 (1995), 308-312.

[5] J. Dixmier, $C^{*}$-algebras, North-Holland Math. Library 15 (North-Holland, Amsterdam, 1982).

[6] G. B. Folland, A course in abstract harmonic analysis, Studies in Advanced Mathematics (CRC Press, Boca Raton, FL, 1995).

[7] P. de la Harpe and A. Valette, La propriété (T) de Kazhdan pour les groupes localement compacts, Astérisque 175 (Société Mathématique de France, 1989).

[8] D. A. Kazhdan, 'Connection of the dual space of a group with the structure of its close subgroups', Funct. Anal. Appl. 1 (1967), 63-65.

[9] A. Lubotzky, Discrete groups, expanding graphs and invariant measures, Progress in Mathematics 125 (Birkhäuser, Basel, 1994).

[10] A. Lubotzky and I. Pak, 'The product replacement algorithm and Kazhdan's property (T)', J. Amer. Math. Soc. 14 (2000), 347-363.

[11] M. Neuhauser, 'Kazhdan constants for conjugacy classes of compact groups', J. Algebra 270 (2003), 564-582.

[12] I. Pak and A. Żuk, 'On Kazhdan constants and mixing of random walks', Int. Math. Res. Not. 36 (2002), 1891-1905.

\section{Markus Neuhauser}

Department of Mathematics C

TU Graz

Steyrergasse 30 / III

8010 Graz

Austria

e-mail: neuhauser@ finanz.math.tu-graz.ac.aî 